\title{
Raman Spectroscopic Studies on the Interaction between Counterions and Polyphosphate Ion
}

\author{
Shinobu Koda, Teruyuki KawaKami, and Hiroyasu Nomura \\ Department of Chemical Engineering, School of Engineering, \\ Nagoya University, Chikusa-ku, Nagoya, 464-01, Japan
}

(Received August 12, 1993)

\begin{abstract}
Interactions between polyphosphate ions and several cations, such as $\mathrm{Na}^{+}, \mathrm{Cs}^{+}$, $\mathrm{Ca}^{2+}$, and $\mathrm{Ba}^{2+}$ were investigated by means of Raman spectroscopy. A Raman band assigned to the $\mathrm{PO}$ vibrational stretching mode of polyphosphate neutralized by $\left(\mathrm{CH}_{3}\right)_{4} \mathrm{NOH}$ in solution shifted to the higher frequency side with increasing concentration of added cations, but no frequency shift was observed in $\mathrm{NaH}_{2} \mathrm{PO}_{4}$ solution. The magnitude of the frequency shift is in the order of $\mathrm{Ca}^{2+}$, $\mathrm{Ba}^{2+}, \mathrm{Na}^{+}$, and $\mathrm{Cs}^{+}$. The Raman intensity varied with the addition of the cations. However, the addition of tetramethylammonium salt did not affect the PO stretching mode of polyphosphate. It is concluded from these results that the counterions closely approach polyions so as to perturb the force constant and the polarizability derivative of the PO bonds and interaction between polyphosphate ion and cations is not purely covalent.
\end{abstract}

KEY WORDS Raman Spectroscopy / Ion Binding / Polyphosphate /

Interactions between biopolymer and metal ions are important factors to determine the physico-chemical properties and functionality of biopolymers. As deoxyribonucleic acid (DNA) molecules contain charged phosphate groups, the investigation of polyphosphate in solutions gives useful information to interpret interactions between DNA and metal cations.

The interaction between polyphosphate ion and counterion has been investigated from the viewpoint of ion binding in polyelectrolyte solutions. Early electrophoretic, viscosity, solubility and dilatometric studies on polyphosphate solution were reported by Strauss and co-workers. ${ }^{1-3}$ Dilatometric and ultrasonic studies were carried out by Spegt and Weill ${ }^{4,5}$ and Zana and Tondre, ${ }^{6,7}$ respectively. These results were interpreted in terms of specific interactions between polyphosphate ion and counterions, sometimes named as the site binding, or ion condensation. Ion-binding states due to the interaction are roughly classified into atmosphere ion binding and specific interactions, which lead to hydration volume changes around ions and/or to complex formation between polyion and counterions. In previous papers, ${ }^{8-10}$ we indicated that sound velocity and adiabatic compressibility of polyelectrolyte solutions are very sensitive to change of hydration volume around ions and $30 \%$ of sodium ions bind to polyphosphate ions. No Raman spectroscopic study was given. Spectroscopic data are useful to obtain the details of interactions and structure of the interaction site.

Raman spectroscopy is useful to study change of local structure and the intra- and intermolecular interaction. The dissociation behavior of some polyelectrolytes was investigated by Lapanje and Rice, ${ }^{11}$ and Tanaka $e t$ al. ${ }^{12}$ In previous papers, ${ }^{13,14}$ we confirmed that the nature of the interaction between polyacrylate ion and alkali metal cations is simply electrostatic and in the case of several divalent cations, such as $\mathrm{Cu}^{2+}, \mathrm{Zn}^{2+}$, and $\mathrm{Mn}^{2+}$, it includes a specific interaction with bond for- 
mation. Interaction between polyphosphate ions and cations, such as $\mathrm{Na}^{+}, \mathrm{Cs}^{+}, \mathrm{Ca}^{2+}$, and $\mathrm{Ba}^{2+}$ was investigated, using the Raman spectrometer. The purpose of this work is to elucidate the nature of interactions between polyphosphate ion and counterions from spectroscopic data. We concentrate our attention on the change of the Raman band frequency of the PO stretching mode in polyphosphate ion, since changes in Raman spectra are observed if interaction between polyion and counterions is strong.

\section{EXPERIMENTAL}

\section{Samples}

Sodium polyphosphate (NaPP) was synthesized following the method given by Strauss et al. ${ }^{1}$ The molecular weight of the sample was $1.3 \times 10^{4}$, as determined from viscosity measurement. Tetramethylammonium polyphosphate (TMAPP) was obtained by stirring with a TMA-neutralized cation exchange resin and passing the solution through a column of TMA-resin. The details of the sample preparation method are given in the literature. ${ }^{6}$ TMACl, $\mathrm{NaCl}, \mathrm{CsCl}, \mathrm{BaCl}_{2}$, and $\mathrm{CaCl}_{2}$ were of guaranteed reagent grade and used without further purification. All aqueous solutions were prepared using deionized water.

\section{Measurement}

Raman spectra of polyphosphate solutions were recorded with a laser Raman spectrometer NR-1800 (Japan Spectroscopic Co., Ltd.) at room temperature. An argon ion laser (NEC GLG 3300), operated at $488 \mathrm{~nm}(300 \mathrm{~mW})$, was used as the light source. Raman spectra of TMAPP solution were measured as a function of concentrations of added salts. The concentration of TMAPP was 0.1 monomer $\mathrm{mol} \cdot 1^{-1}$.

\section{RESULTS AND DISCUSSION}

Zwicket et $a l^{15}$ clarified the ionization behavior of phosphoric acid from Raman spectroscopic study. Several Raman bands attributed to different states of the ionization of phosphoric acid were observed in a frequency range from 800 to $1200 \mathrm{~cm}^{-1}$. In order to assign the Raman bands of polyphosphate, the Raman spectrum of $\mathrm{NaH}_{2} \mathrm{PO}_{4}$ in solution was measured. Raman bands characteristic of $\mathrm{H}_{2} \mathrm{PO}_{4}{ }^{-}$were at 879 and $1077 \mathrm{~cm}^{-1}$. These two Raman bands are not affected by $\mathrm{NaCl}$.

Figure 1 shows the Raman spectra of TMAPP as a function of added $\mathrm{NaCl}$ concentration. Polyphosphate neutralized by $\mathrm{TMA}^{+}$ion is used as a reference state of polyphosphate ion. As tetramethylammonium ion is bulky and the interaction between tetramethylammonium ion and polyphosphate ion is very weak, the effect of tetramethylammonium ion on polyphosphate ion is negligibly small. No new Raman bands due to the metal-oxygen bond were observed in the frequency range from 300 to $800 \mathrm{~cm}^{-1}$. This means that covalent metal-oxygen bonds are not formed in solutions of polyphosphate salts and interaction between cations and polyphosphate ion is not purely covalent. The Raman band observed at $855 \mathrm{~cm}^{-1}$ was assigned to the $\mathrm{CN}$ stretching mode in tetra-

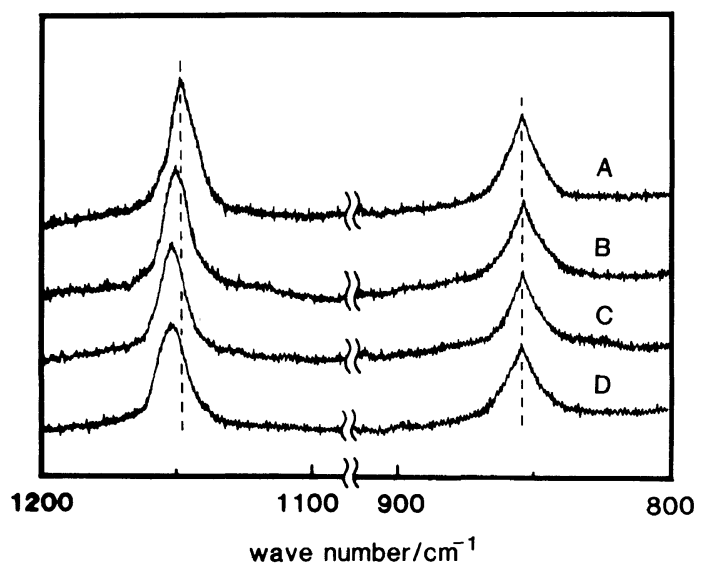

Figure 1. Raman spectra of TMAPP in aqueous solution as a function of $\mathrm{NaCl}$ concentration. $\mathrm{NaCl}$ concn (mol . $1^{-1}$ ): 0 (A), 0.2 (B), 0.4 (C), 0.6 (D). 


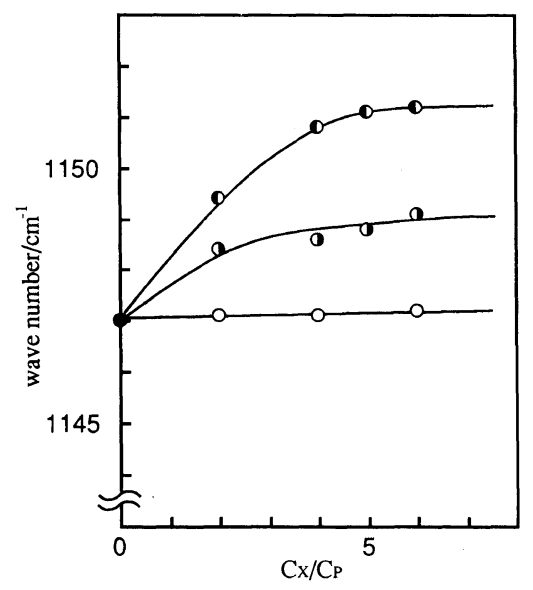

Figure 2. Vibrational frequency of the PO stretching mode against the $\mathrm{C}_{\mathrm{x}} / C_{\mathrm{p}}$. $C_{\mathrm{x}}$, concentration of added salt and $C_{\mathrm{p}}$, concentration per monomer unit of TMAPP. , without salt; $\mathbf{O}, \mathrm{NaCl} ; \mathrm{O}, \mathrm{CsCl}$;, $\mathrm{TMACl}$.

methyl ammonium ion. The Raman band at $855 \mathrm{~cm}^{-1}$ was used as an internal reference band for central frequency and the band intensity. In polyphosphate solution, only one Raman band due to the $-\mathrm{PO}_{3}{ }^{-}$unit was observed in the frequency range from 800 to $1200 \mathrm{~cm}^{-1}$. The Raman band located at about $1150 \mathrm{~cm}^{-1}$ was assigned to the PO stretching mode of polyphosphate ion. The central frequency shifts to the higher frequency side with increasing $\mathrm{NaCl}$ concentration and approaches a certain constant value. The change of the central frequency is plotted against the added salt concentration in Figure 2. The addition of $\mathrm{CsCl}$ to TMAPP solution causes a slight frequency shift in comparison with addition of $\mathrm{NaCl}$. No frequency shift was observed when TMACl was added to TMAPP solution, since the interaction between $\mathrm{TMA}^{+}$ ion and $\mathrm{PP}^{-}$ion was weak, as mentioned above.

The Raman spectra of polyphosphate ion in divalent salt solution are shown in Figure 3. The addition of large amount of divalent salts into TMAPP solution results in precipitation. Therefore, the Raman spectra were measured at the low salt concentrations before the onset of precipitation. As in the case of the addition

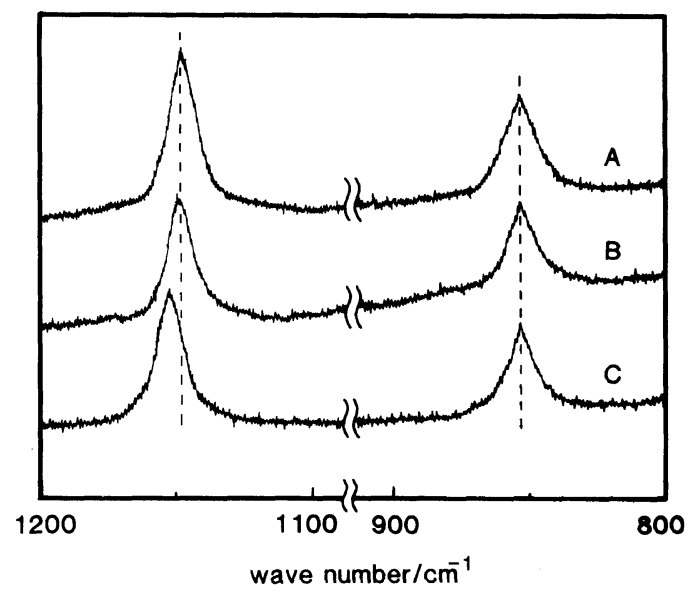

Figure 3. Raman spectra of TMAPP in aqueous solutions. (A) salt-free solution; (B) $0.01 \mathrm{~mol} \cdot 1^{-1} \mathrm{BaCl}_{2}$; (C) $0.012 \mathrm{~mol} \cdot 1^{-1} \mathrm{CaCl}_{2}$.

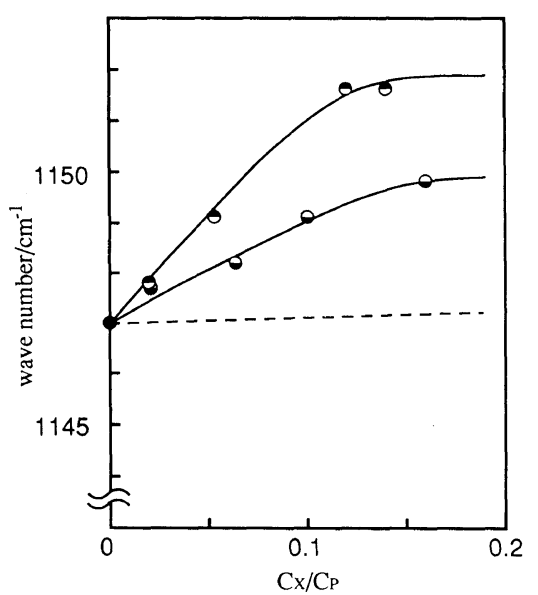

Figure 4. Vibrational frequency of $\mathrm{PO}$ stretching mode against $C_{\mathrm{x}} / C_{\mathrm{p}}$. The broken line: $\mathrm{NaCl}, \boldsymbol{\odot}$, without salt; $\ominus$, $\mathrm{CaCl}_{2} ; \ominus, \mathrm{BaCl}_{2}$.

of monovalent salt, no new Raman bands due to the metal-oxygen bond formation were observed and only the Raman band due to the PO stretching vibrational mode changed. The change of the central frequency of the PO stretching mode was shown in Figure 4. Large frequency shift was observed at the low concentration range, where the vibrational frequency of the PO stretching mode was almost independent of $\mathrm{NaCl}$ concentration. In polyacrylate solution, the Raman band located 
at $1412 \mathrm{~cm}^{-1}$, assigned to the vibrational mode of $\mathrm{COO}^{-}$group, shifted to the higher frequency side with addition of $\mathrm{Cu}^{2+}, \mathrm{Mn}^{2+}$, and $\mathrm{Zn}^{2+}$ into polyacrylate solution. Thus, the higher frequency shift is recognized in Raman bands due to the $\mathrm{PO}^{-}$and $\mathrm{COO}^{-}$groups in polyelectrolytes. In simple electrolyte solutions, no frequency shift was observed, that is, the frequency change was characteristic of polyphosphate solution not but to $\mathrm{NaH}_{2} \mathrm{PO}_{4}$ solution. The magnitude of the frequency shift depended on cation species and their concentrations, and was in the order of $\mathrm{Ca}^{2+}>$ $\mathrm{Ba}^{2+}>\mathrm{Na}^{+}>\mathrm{Cs}^{+}>\mathrm{TMA}^{+}$, as shown in Figures 2 and 4 . With increase of the charge density of added cations, PO stretching vibrational mode was more perturbed by added cations. The frequency change was caused by the reduction of the electrostatic field due to the ion-binding.

In general, the classical theory of the vibration shows that the vibrational frequency (v) can be calculated with reduced mass $(M)$ and force constant $(k)$, such as $v \propto \sqrt{k / M}$. Thus, the higher frequency shift is caused by the increase of $k$ or decrease of $M$. The reduced mass of PO stretching vibrational mode in the $\mathrm{P}-\mathrm{O} \cdot \mathrm{M}$ state was of the same magnitude or larger than that of the free or dissociated PO state. Change of the reduced mass caused shift to the lower frequency side. The main factor of the observed frequency shift was change of the force constant of PO bond, that is, the second order derivative of the interatomic potential at the equilibrium distance. The PO bond in the $\mathrm{P}-\mathrm{O} \cdot \mathrm{M}$ state vibrates in a weaker electrostatic field than that in the non-binding state, since the degree of the ionization of the PO bond is reduced by counterion binding.

The band intensity was affected by the added salt. Figures 5 and 6 show the normalized intensity as a function of concentration of added salt. The band intensity decreased with increasing salt concentration. The small amount of the divalent salt resulted in large decrease of the intensity in comparison with

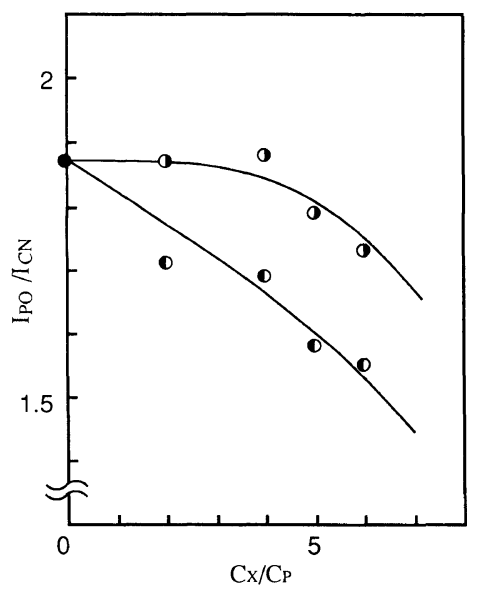

Figure 5. Plot of normalized intensity of the PO stretch-

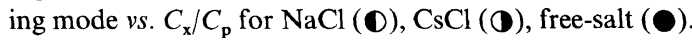

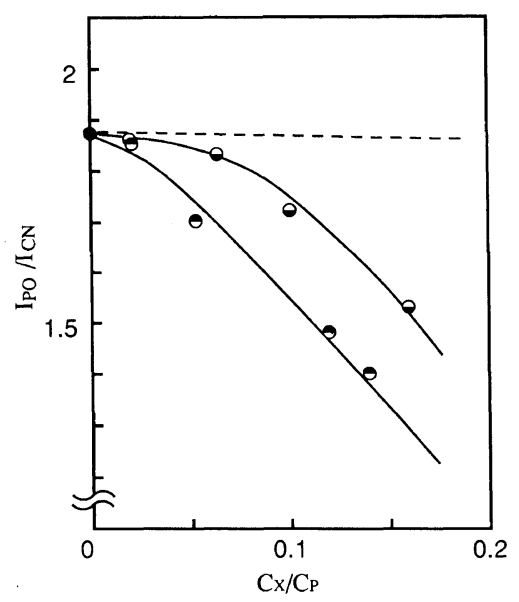

Figure 6. Plot of normalized intensity of the $\mathrm{PO}$ stretching mode $v s . C_{\mathrm{x}} / C_{\mathrm{p}}$ for $\mathrm{NaCl}$ (the broken line), $\mathrm{BaCl}_{2}(\Theta)$ and $\mathrm{CaCl}_{2}(\Theta)$.

the case of monovalent salt. The Raman intensity was proportional to the number of molecules and bond polarizability derivative of interest. The decrease of the band intensity means that the counterion binding reduces the bond polarizability derivative.

In previous paper, ${ }^{16}$ we obtained the degree of ion binding on polyphosphate ion by measurement of the sound velocity or the adiabatic compressibility. The degree of ion binding was sensitive to the hydration volume 
change due to the strong interaction between polyphosphate ion and counterion. The degree of ion binding thus obtained was $0.25-0.3$. Strauss and co-workers reported the degree of ion binding below 0.5 by electrophoretic ${ }^{2}$ and dilatometric $^{3}$ studies. Based on the relaxation rate of $\mathrm{Co}^{2+}$ determined in the NMR study, Spegt et al. indicated that the counterion of $c a$. $40 \%$ penetrates the hydration layer around polyphosphate ion. ${ }^{4,5}$ The degree of ion binding estimated from the sound velocity data was slightly smaller than those indicated above. Though the interaction due to the ion binding does not give rise to the new Raman band, counterions approach polyion closely so as to disturb the $\mathrm{PO}^{-}$stretching vibration.

Acknowledgement. The present work was supported in part by a Grant-in-Aid for Scientific Research (No. 04215102) on Priority Area of "Molecular Approaches to Nonequilibrium Processes in Solutions" from the Ministry of Education, Science, and Culture of Japan.

\section{REFERENCES}

1. U. P. Strauss, E. H. Smith, and P. L. Wineman, J. Am. Chem. Soc., 75, 3959 (1953).

2. U. P. Strauss and P. D. Ross, J. Am. Chem. Soc., 81, 5295 (1959).

3. U. P. Strauss and Y. P. Leung, J. Am. Chem. Soc., 87, 1476 (1965).

4. P. Spegt and G. Weill, C. R. Acad. Sci. (Paris), 274, 587 (1972).

5. P. Spegt and G. Weill, Biophys. Chem., 4, 143(1976).

6. C. Tondre and R. Zana, J. Phys. Chem., 75, 3367 (1971).

7. R. Zana and C. Tondre, Biophys. Chem., 1, 367 (1974).

8. S. Koda, H. Nomura, and M. Nagasawa, Biophys. Chem., 23, 147 (1985).

9. S. Koda, S. Tsukamoto, and H. Nomura, Biophys. Chem., 28, 115 (1985).

10. S. Koda, T. Kitano, S. Kawaguchi, and H. Nomura, Rep. Prog. Polym. Phys. Jpn., 29, 41 (1986).

11. S. Lapanje and S. A. Rice, J. Am. Chem. Soc., 83, 496 (1961).

12. N. Tanaka, H. Kitano, and N. Ise, Macromolecules, 22, 2652 (1989).

13. S. Koda, H. Nomura, and M. Nagasawa, Biophys. Chem., 15, 65 (1982).

14. S. Koda, H. Nomura, and M. Nagasawa, Biophys. Chem., 18, 361 (1983).

15. A. Zwick, F. Lakhdar-Ghazal, and J.-F. Tocanne, $J$. Chem. Soc., Faraday Trans., 2, 85, 783 (1989).

16. S. Koda and H. Nomura, Rep. Prog. Polym. Phys. Jpn., 29, 41 (1986). 